Research Article

\title{
In Search of Risk Factors for Recurrent Erysipelas and Cellulitis of the Lower Limb: A Cross-Sectional Study of Epidemiological Characteristics of Patients Hospitalized due to Skin and Soft-Tissue Infections
}

\author{
Mariusz Sapuła (D), Dagny Krankowska (D), and Alicja Wiercińska-Drapało \\ Department of Infectious and Tropical Diseases and Hepatology, Medical University of Warsaw, Hospital for Infectious Diseases, \\ Warsaw, Poland \\ Correspondence should be addressed to Dagny Krankowska; d.krankowska@gmail.com
}

Received 2 March 2020; Accepted 16 April 2020; Published 7 May 2020

Academic Editor: Diamantis Kofteridis

Copyright (c) 2020 Mariusz Sapuła et al. This is an open access article distributed under the Creative Commons Attribution License, which permits unrestricted use, distribution, and reproduction in any medium, provided the original work is properly cited.

\begin{abstract}
Background. Erysipelas and cellulitis are common, acute, bacterial infections of the skin and subcutaneous tissue. The incidence of these infections is growing, and the recurrence rate is high. Effective antibiotic prophylaxis is available, but insufficient data exist on the risks factors for recurrent infection. Purpose. To compare comorbidities and laboratory findings in patients with singleepisode and recurrent erysipelas/cellulitis in order to identify risk factors for recurrent erysipelas/cellulitis. Methods. A crosssectional study, which included patients hospitalized in the Department of Infectious and Tropical Diseases and Hepatology of the Medical University of Warsaw due to erysipelas and cellulitis during 3 consecutive years (July 2016-June 2019). Results. The study included 163 patients, of which 98 had a first episode of erysipelas/cellulitis and 65 had a recurrence. The recurrent infection was significantly associated with a history of lymphedema (12.3\% in the recurrent group vs. $2.0 \%$ in the first-episode group, $p=0.015$ ), a higher BMI (35.4 vs. 31.2 , respectively, $p=0.002)$, chronic obstructive pulmonary disease $(10.8 \%$ vs. $2.0 \%, p=0.030)$, and a shorter history of symptoms prior to hospitalization ( 6.0 days vs. 11.8 days, $p=0.004$ ). Patients with the first episode of infection were more likely to have had minor local trauma directly preceding the symptoms of infection ( $20.4 \%$ in the first-episode group vs. $1.5 \%$ in the recurrent group, $p=0.001$ ). Conclusions. Patients with lymphedema and obesity should be viewed at high risk of developing recurrence of erysipelas and thus should be considered as candidates for antibiotic prophylaxis and other prevention methods. Minor local trauma directly preceding the skin infection does not by itself confer a higher risk for erysipelas recurrence. More research is needed to assess the association of recurrent skin and soft-tissue infection to preceding minor local trauma, individual components of the metabolic syndrome, and COPD.
\end{abstract}

\section{Introduction}

Erysipelas and cellulitis are common, acute, bacterial infections of the skin and underlying soft tissue. Traditionally, erysipelas was distinguished from cellulitis by a more distinct, elevated border and it was presumed to be different in etiology, but recently this distinction has been put into question by many authors [1-3].

It is frequently assumed that erysipelas and cellulitis are caused by group A streptococci and Staphylococcus aureus, but other streptococci and Gram-negative bacteria seem to be the causative agents in some cases [2-4]. These infections usually affect the lower limb and can be diagnosed clinically in patients presenting with sudden onset of pain, edema, erythema, and increased warmth of affected localization, as well as systemic symptoms such as fever and chills. Bullae may also appear on the skin. Laboratory findings are nonspecific and may involve increased white blood cell count with a predominance of neutrophils and an elevated $\mathrm{C}$-reactive protein. 
Some studies show an increase in the incidence of erysipelas and cellulitis, and in European countries, it is estimated to be 200 per 100,000 people per year [5].

Various risk factors for the initial episode of erysipelas and cellulitis of the lower limb were found in previous studies: local edema/lymphedema, venous insufficiency, disruption of the cutaneous barrier, and being overweight $[6,7]$.

Patients with these infections can usually be successfully treated with narrow-spectrum penicillins, but sometimes require hospitalization and intravenous treatment. The most common complication of erysipelas is recurrence of the infection. The recurrence occurs in up to $41 \%$ of patients in 5 years [8].

A number of interventional studies focused on pharmacological prophylaxis of recurrence. Most of these included patients with at least two episodes of infection in the span of 3 years and evaluated narrow-spectrum antibiotics, mainly penicillin, which seemed to be effective for the duration of the prophylaxis [9]. Some demonstrable causes of penicillin prophylaxis failure include noncompliance and erysipelas due to resistant pathogens (such as MRSA or Gram-negative bacteria); incorrect dose and selecting an antibiotic without proven efficacy in this setting are other possible causes enumerated by Koster et al. [10].

Although effective antibiotic prophylaxis for recurrent prophylaxis is available, insufficient data exist on the risks factors for recurrent infection, and so it may be difficult to predict whether a given patient is a good candidate for such interventions.

\section{Aim}

To compare demographic characteristics, comorbidities, and laboratory findings in patients with first-episode erysipelas and recurrent erysipelas in order to identify risk factors for recurrent erysipelas.

\section{Materials and Methods}

Medical records of patients hospitalized in the Department of Infectious and Tropical Diseases and Hepatology of the Medical University of Warsaw, Poland, due to erysipelas or cellulitis during 3 consecutive years (July 2016-June 2019) were analyzed in the study. For the purposes of this study, no distinction was made between patients with erysipelas and cellulitis (henceforth referred to collectively simply as "erysipelas") as there was no difference in the management of this condition in our clinical center. Patients with erythematous and bullous erysipelas were included in the study, but those with necrotizing fasciitis and osteomyelitis were excluded. Only patients with erysipelas of the lower limb were included.

Demographic, clinical, and laboratory markers were recorded into a database, and the data were analyzed using statistical software to compare patients with first-episode and recurrent erysipelas.

If more than one record of a recurrent episode of a particular patient was present in the database, only the first one was included into the analysis.
For data analysis, the chi-squared test (or Fisher's exact test, if applicable) and Student's $t$-test were used in a univariate analysis to compare the qualitative and quantitative variables between groups. The $R$ statistical analysis software was used for all calculations.

\section{Results}

4.1. Sample Baseline Characteristics. A total of 163 patients hospitalized with erysipelas of the lower extremity were identified during 3 consecutive years (July 2016-June 2019). The mean age was 66.1 years (median age was 65 years), and $47.2 \%$ were female. There were 98 patients with single-episode erysipelas (SE) and 65 patients with recurrent erysipelas (RE). The median number of episodes for the recurrent erysipelas group was 3 , ranged from 2 to more than 10 . Table 1 shows the distribution of the number of erysipelas episodes.

Blood cultures on admission were performed in 40 patients (24.5\% of total) and were positive in 2 patients $(5.0 \%$ of tested patients)—one culture yielded Streptococcus pyogenes and the other one Streptococcus sp. pertaining to group C.

5 patients from the recurrent group $(7.7 \%)$ were on antibiotic erysipelas prophylaxis at the time of recurrence.

Only one patient was found to have concomitant deep venous thrombosis of the affected limb and one patient developed a minor skin abscess, although ultrasound of the affected limb was performed only when clinically indicated (22.3\% of cases).

4.2. Comparison between Single-Episode Erysipelas and Recurrent Erysipelas. There was no difference between the age $(p=0.076)$ nor gender distribution $(p=0.386)$ between the two groups (Table 2). Patients with RE had a significantly higher body mass index (BMI) (35.4 vs. 31.2; $p=0.002)$, and they were more likely to have a history of lymphedema ( $12.3 \%$ vs. $2.0 \% ; p=0.015)$ as well as residual lymphedema at discharge $(15.4 \%$ vs. $5.1 \% ; p=0.026)$ than patients with SE (Tables 3 and 4). The only chronic illness that showed a significant association with RE was chronic obstructive pulmonary disease (COPD) (10.8\% vs $2.0 \%$; $p=0.030$ ) (Table 3).

Trauma preceding the development of erysipelas was much more common in SE than in RE (10.8\% vs. $2.0 \%$; $p=0.030$ ) (Table 4).

Laboratory findings were compared between patients with SE and RE, but no significant differences were observed (Table 5). The length of stay in the hospital was similar for both groups. Patients who had the first episode of erysipelas had a longer history of symptoms preceding hospital admission in comparison with those who had a recurrent episode (11.8 days vs. 6.0 days; $p=0.004)$ (Table 6).

As lymphedema can lead to confounding when comparing the BMI of both groups, a separate comparison was done with a subgroup of patients without a history of lymphedema (Table 7). No inferential statistics were done on the subgroup with a history of lymphedema, as the number of cases in this subgroup was too small $(n=10)$. 
TABLe 1: The distribution of the number of erysipelas episodes for the recurrent group.

\begin{tabular}{|c|c|c|c|c|c|c|c|c|c|}
\hline Number of episodes & 2 & 3 & 4 & 5 & 6 & 7 & 8 & 9 & $\geq 10$ \\
\hline Number of patients (percentage of patients) & $23(35 \%)$ & $10(15 \%)$ & $10(15 \%)$ & $12(19 \%)$ & $2(3 \%)$ & $2(3 \%)$ & $1(2 \%)$ & $0(0 \%)$ & $5(8 \%)$ \\
\hline
\end{tabular}

TABLE 2: Association of demographic characteristics and recurrent lower extremity erysipelas.

\begin{tabular}{lccr}
\hline Parameter & First episode & Recurrent episode & $p$ \\
\hline Gender, female & $50.0 \%$ & $43.6 \%$ & 0.386 \\
Age (years) & $67.9+/-16.7$ & $63.4+/-14.8$ & 0.076 \\
Homelessness & $4.1 \%$ & $4.6 \%$ & 1.000 \\
\hline
\end{tabular}

Age shown as mean + /- standard deviation.

TABLE 3: Association of comorbidities and recurrent lower extremity erysipelas.

\begin{tabular}{lccc}
\hline Comorbidity & First episode & Recurrent episode & $p$ \\
\hline BMI $\left(\mathrm{kg} / \mathrm{m}^{2}\right)$ & $31.2+/-7.7$ & $35.4+/-8.9$ & 0.002 \\
Hypertension & $69.4 \%$ & $33.8 \%$ & 0.983 \\
Diabetes mellitus & $26.5 \%$ & $24.6 \%$ & 0.316 \\
Dyslipidemia & $28.6 \%$ & $33.8 \%$ & 0.577 \\
Congestive heart failure & $32.7 \%$ & $9.2 \%$ & 0.874 \\
Chronic kidney disease & $12.2 \%$ & $10.8 \%$ & 0.548 \\
Chronic obstructive pulmonary disease & $2.0 \%$ & $1.5 \%$ & 0.030 \\
Immunodeficiency & $9.1 \%$ & $4.6 \%$ & 0.052 \\
Cancer, history & $8.2 \%$ & $0.2 \%$ & 0.528 \\
Cancer, present & $0.5 \%$ & $4.6 \%$ & 0.404 \\
History of stroke & $5.1 \%$ & $3.1 \%$ & 1.000 \\
History of myocardial infarction & $7.1 \%$ & & 0.319 \\
\hline
\end{tabular}

Quantitative variables are shown as mean $+/$-standard deviation.

TABLE 4: Association of local risk factors and recurrent lower extremity erysipelas.

\begin{tabular}{lccc}
\hline Local risk factor & First episode & Recurrent episode & $p$ \\
\hline Chronic ulcer & $9.2 \%$ & $15.4 \%$ & 0.227 \\
Chronic dermatological disease & $12.2 \%$ & $7.7 \%$ & 0.352 \\
Minor local trauma & $20.4 \%$ & $1.5 \%$ & 0.001 \\
History of lymphedema & $2.0 \%$ & $12.3 \%$ & 0.015 \\
Lymphedema at discharge & $5.1 \%$ & $15.4 \%$ & 0.026 \\
\hline
\end{tabular}

TABLE 5: Association of laboratory parameters and recurrent lower extremity erysipelas.

\begin{tabular}{lccc}
\hline Parameter & First episode & Recurrent episode & $p$ \\
\hline Hemoglobin $(\mathrm{g} / \mathrm{dL})$ & $12.7+/-1.9$ & $13.1+/-1.4$ & 0.092 \\
Platelet count (G/L) & $228+/-93$ & $243+/-104$ & 0.358 \\
White blood cell count (G/L) & $10.9+/-4.9$ & $10.8+/-4.3$ & 0.885 \\
Neutrophil count (G/L) & $8.3+/-4.7$ & $8.4+/-4.4$ & 0.854 \\
C-reactive protein (mg/l) & $130+/-111$ & $114+/-97$ & 0.335 \\
Procalcitonin (ng/ml) & $2.97+/-7.62$ & $1.20+/-1.83$ & 0.152 \\
D-dimers (ng/ml) & $1618+/-1243$ & $1387+/-1058$ & 0.273 \\
Urea (mmol/l) & $8.3+/-5.5$ & $7.3+/-2.9$ & 0.199 \\
Creatinine at admission (umol/l) & $97+/-67$ & $92+/-47.1$ & 0.598 \\
Creatinine at discharge (umol/l) & $73.9+/-21.5$ & $72.9+/-19.5$ & 0.764 \\
\hline
\end{tabular}

Values are shown as mean $+/$-standard deviation.

\section{Discussion}

In our study, similarly as in Inghammar et al. [11] erysipelas recurrence was significantly associated with increased BMI, but not with other comorbidities associated with the metabolic syndrome (hypertension, diabetes mellitus, or dyslipidemia). In a study by BrishkoskaBoshkovski et al. [3], in addition to an association between obesity (but no differences in mean BMI) and RE, the recurrence was also linked to diabetes mellitus treated with 
TABLE 6: Association of other parameters and recurrent lower extremity erysipelas.

\begin{tabular}{|c|c|c|c|}
\hline Parameter & First episode & Recurrent episode & $p$ \\
\hline Oral antibiotics prior to admission & $39.8 \%$ & $26.2 \%$ & 0.073 \\
\hline Hospitalisation duration (days) & $15.8+/-7.1$ & $14.5+/-6.1$ & 0.205 \\
\hline Symptoms' duration prior to admission (days) & $11.8+/-17.5$ & $6.0+/-7.9$ & 0.004 \\
\hline Bilaterality of erysipelas & $9.2 \%$ & $7.7 \%$ & 0.739 \\
\hline Bullous presentation & $25.5 \%$ & $15.4 \%$ & 0.123 \\
\hline
\end{tabular}

Quantitative variables are shown as mean $+/$ - standard deviation.

TABLE 7: Association of BMI and recurrent lower extremity erysipelas in patients without lymphedema: 96 patients with first episode and 57 patients with recurrence.

\begin{tabular}{lccc}
\hline Parameter & First episode & Recurrent episode & $p$ \\
\hline BMI $\left(\mathrm{kg} / \mathrm{m}^{2}\right)$ & $31.4+/-7.2$ & $34.0+/-8.9$ & 0.035 \\
\hline
\end{tabular}

BMI value is shown as mean $+/-$ standard deviation.

insulin. Obesity and diabetes were also independent predictors of lower limb cellulitis recurrence in a large longitudinal cohort study by Cannon et al. [12]. In a study by Kozłowska et al., being overweight and having hypertension but not diabetes were more prevalent in patients with recurrent erysipelas [13]. On the other hand, Karppelin et al. did not find any association between components of the metabolic syndrome and erysipelas recurrence [8]. The association between the metabolic syndrome and erysipelas needs further research.

Apart from obesity, the only chronic disease that was positively associated with recurrent erysipelas in our study was chronic obstructive pulmonary disease (COPD). This is in contrast to the results of Inghammar et al. where COPD was more prevalent in first-episode erysipelas. Inghammar et al. argued that this might be due to the high use of antibiotics against respiratory infections in this group of patients and that it might cause a decrease of colonizing agents responsible for erysipelas. Cannon et al. also mention chronic pulmonary disease as an independent risk factor for RE, but the authors do not specify which chronic diseases they mean [12].

Some authors stress the importance of toe web dermatophytosis as a risk factor for erysipelas [6, 8, 14]. Due to the retrospective character of our study, certain medical records lacked the information about the presence or absence of toe web intertrigo; thus, we did not include this potential risk factor in the statistical analysis of this study.

Both in this and previous studies, minor trauma was seen mainly in the setting of first-episode erysipelas $[3,8,11,14]$. One possible interpretation is that the disruption of the skin barrier predisposes to a single, nonrecurrent episode of erysipelas, whereas the recurrence of infection is facilitated mainly by chronic risk factors. On the other hand, one episode of erysipelas may itself be a risk factor for a repeated episode of infection, and thus some patients with a recent history of minor trauma could still be at risk for recurrence. Furthermore, research is required to support this hypothesis.

Lymphedema is an established risk factor for RE and frequent complication of erysipelas $[3,8,11,12]$. Our data are consistent with previous studies, as patients with RE were more likely to have a history of lymphedema (12.3\% vs. $2.0 \%$; $p=0.015)$ than patients with SE. As Chlebicki et al. pointed out in their paper, a vicious circle is created with lymphedema being both the complication of erysipelas and an important risk factor for recurrence [15].

We found no differences in the studied laboratory parameters between groups, as opposed to Brishkoska-Boshkovski et al. [16].

There are some limitations in this study. This study was not prospective in nature, and thus there is a possibility of bias: the patients included in the first-episode group might in the future have a recurrence, thus biasing the result in the direction of possibly missing a statistically significant association. Another possible limitation is the fact that the studied population was comprised of hospitalized patients, and thus the conclusions of this study may not be applicable to patients with erysipelas treated in the outpatient clinic. In spite of the above, we believe that the study design and its conclusions are appropriate and valid given the clinical problem that we aimed to explore in this study.

\section{Conclusions}

Patients with lymphedema and obesity should be viewed at high risk of developing recurrence of erysipelas and thus should be considered as candidates for antibiotic prophylaxis and other prevention methods. Minor local trauma directly preceding the skin infection does not by itself confer a higher risk for erysipelas recurrence. More research is needed to assess the association of preceding minor local trauma, individual components of the metabolic syndrome, and COPD to recurrent skin and soft-tissue infection.

\section{Data Availability}

The data generated and/or analyzed during the current study are available from the author at mariusz.sapula@gmail.com upon reasonable request.

\section{Ethical Approval}

The study was conducted according to the international (Helsinki Declaration), national, and local (Medical University of Warsaw, Poland) bioethical guidelines and regulations. As no procedures other that standard of care and anonymized data analysis were performed during the study, no additional ethics committee approval was necessary. 


\section{Consent}

In this study, data regarding previous hospitalizations were extracted from hospital databases, anonymized, and subsequently analyzed using statistical software. No procedures other than standard of care were performed on the patients. Informed consent was therefore not applicable.

\section{Conflicts of Interest}

The authors declare no conflicts of interest.

\section{References}

[1] C. G. Gunderson and R. A. Martinello, "A systematic review of bacteremias in cellulitis and erysipelas," Journal of Infection, vol. 64, no. 2, pp. 148-155, 2012.

[2] D. R. Cranendonk, A. P. M. Lavrijsen, J. M. Prins, and W. J. Wiersinga, "Cellulitis: current insights into pathophysiology and clinical management," The Netherlands Journal of Medicine, vol. 75, no. 9, pp. 366-378, 2017.

[3] V. Brishkoska-Boshkovski, I. Kondova-Topuzovska, K. Damevska, and A. Petrov, "Comorbidities as risk factors for acute and recurrent erysipelas," Open Access Macedonian Journal of Medical Sciences, vol. 7, no. 6, pp. 937-942, 2019.

[4] B. Eriksson, C. Jorup-Ronstrom, K. Karkkonen, A. C. Sjoblom, and S. E. Holm, "Erysipelas: clinical and bacteriologic spectrum and serological aspects," Clinical Infectious Diseases, vol. 23, no. 5, pp. 1091-1098, 1996.

[5] S. Bartholomeeusen, J. Vandenbroucke, C. Truyers, and F. Buntinx, "Epidemiology and comorbidity of erysipelas in primary care," Dermatology, vol. 215, no. 2, pp. 118-122, 2007.

[6] A. Dupuy, H. Benchikhi, J.-C. Roujeau et al., "factors for erysipelas of the leg (cellulitis): case-control study," British Medical Journal, vol. 318, no. 7198, pp. 1591-1594, 1999.

[7] S. Bjornsdottir, M. Gottfredsson, A. S. Thorisdottir et al., "Risk factors for acute cellulitis of the lower limb: a prospective casecontrol study," Clinical Infectious Diseases, vol. 41, no. 10, pp. 1416-1422, 2005.

[8] M. Karppelin, T. Siljander, J. Aittoniemi et al., "Predictors of recurrent cellulitis in five years. Clinical risk factors and the role of PTX3 and CRP," Journal of Infection, vol. 70, no. 5, pp. 467-473, 2015.

[9] A. Dalal, M. Eskin-Schwartz, D. Mimouni et al., "Interventions for the prevention of recurrent erysipelas and cellulitis," Cochrane Database of Systematic Reviews, vol. 6, Article ID CD009758, 2017.

[10] J. B. Koster, B. J. Kullberg, and J. W. Van Der Meer, "Recurrent erysipelas despite antibiotic prophylaxis: an analysis from case studies," The Netherlands Journal of Medicine, vol. 65, no. 3, pp. 89-94, 2007.

[11] M. Inghammar, M. Rasmussen, and A. Linder, "Recurrent erysipelas-risk factors and clinical presentation," BMC Infectious Diseases, vol. 14, no. 1, p. 270, 2014.

[12] J. Cannon, J. Dyer, J. Carapetis, and L. Manning, "Epidemiology and risk factors for recurrent severe lower limb cellulitis: a longitudinal cohort study," Clinical Microbiology and Infection, vol. 24, no. 10, pp. 1084-1088, 2018.

[13] D. Kozłowska, H. Myśliwiec, P. Kiluk, A. Baran, A. J. Milewska, and I. Flisiak, "Clinical and epidemiological assessment of patients hospitalized for primary and recurrent erysipelas," Przeglad epidemiologiczny, vol. 70, no. 4, pp. 575-584, 2016.
[14] F. Pavlotsky, S. Amrani, and H. Trau, "Recurrent erysipelas: risk factors. Risikofaktoren Fur Rezidiverysipele," Journal der Deutschen Dermatologischen Gesellschaft, vol. 2, no. 2, pp. 89-95, 2004.

[15] M. P. Chlebicki and C. C. Oh, "Recurrent cellulitis: risk factors, etiology, pathogenesis and treatment," Current Infectious Disease Reports, vol. 16, no. 9, p. 422, 2014.

[16] V. Brishkoska-Boshkovski, I. Dimitrovska, and I. KondovaTopuzovska, "Clinical presentation and laboratory characteristics in acute and recurrent erysipelas," Open Access Macedonian Journal of Medical Sciences, vol. 7, no. 5, pp. 771-774, 2019. 